\title{
A lattice Boltzmann study of phase separation in liquid-vapor systems with gravity
}

\author{
Artur Cristea ${ }^{1}$, Giuseppe Gonnella ${ }^{2}$, Antonio Lamura ${ }^{3}$ and \\ Victor Sofonea ${ }^{1, *}$ \\ ${ }^{1}$ Center for Fundamental and Advanced Technical Research, Romanian Academy, \\ Bd. Mihai Viteazul 24, 300223 Timişoara, Romania \\ 2 Dipartimento di Fisica, Università di Bari and INFN, Sezione di Bari, Via Amendola \\ 173, 70126 Bari, Italy \\ ${ }^{3}$ Istituto Applicazioni Calcolo, CNR, Via Amendola 122/D, 70126 Bari, Italy
}

\begin{abstract}
Phase separation of a two-dimensional van der Waals fluid subject to a gravitational force is studied by numerical simulations based on lattice Boltzmann methods (LBM) implemented with a finite difference scheme. A growth exponent $\alpha=1$ is measured in the direction of the external force.
\end{abstract}

PACS: 47.11.-j, 47.20.Hw, 05.70.Ln

Key words: Lattice Boltzmann, phase separation, liquid-vapor, gravity.

\section{Introduction}

Phase ordering in fluids is an important process that still needs to be completely understood in many cases of practical relevance. When a fluid is quenched from an initial disordered state into a regime of two-phase coexistence below the spinodal line, domains of the two phases are formed and grow with time. The typical size $R$ of domains follows the power law $R \sim t^{\alpha}$ with the growth exponent $\alpha$ being universal in the sense that it does not depend on the microscopic details of the fluid, assuming only a few values related to the physical mechanism operating during phase separation [1]. Hydrodynamics is in general relevant and the coupling with the velocity field can change the value of the growth exponent $\alpha$ from that of purely diffusive growth [2,3].

In this paper we consider the ordering of a liquid-vapor system subject to an external field mimicking the effects of gravity. The role of gravity on phase ordering has been more studied in binary systems. In critical quenches, after an initial diffusive growth

*Corresponding author. Email addresses: f1astra@acad-tim.tm.edu.ro (A. Cristea), gonnella@ba.infn.it (G. Gonnella), a. lamura@ba.iac.cnr.it (A. Lamura), sofonea@acad-tim.tm.edu.ro (V. Sofonea) 
with exponent $1 / 3$, there is a viscous growth characterized by $\alpha=1$ followed by an inertial regime with $\alpha=2 / 3$ [4]. Gravity becomes relevant when heavy domains resting on top of light ones become gravitationally unstable, thus accelerating the domain growth [5]. This occurs at late stages making inertial growth difficult to observe. A theoretical analysis neglecting hydrodynamical contributions suggests an exponent $\alpha_{z}=1$ for the size of domains in the vertical direction [6]. There are few studies of phase separation for liquid-vapor systems. In two-dimensional simulations the values $\alpha=1 / 2$ for high viscosity fluids and $\alpha=2 / 3$ for low-viscosity fluids have been found [2,7]. We are not aware of simulations made on a liquid-vapor system subject to gravity, where the growth exponent is measured.

We address this problem by applying the lattice Boltzmann method (LBM) to simulate a van der Waals fluid described by the Navier-Stokes and the continuity equation. LBM have been proved successful in studying fluids with mesoscopic structures (liquid-vapor interfaces in our case) on large time scales, as it is needed for phase separation [2,4,8-12]. In our approach, the thermodynamic description is based on a free-energy functional where interfaces are described at a coarse-grained level. The free-energy interface cost is expressed, as usual in van der Waals-Landau models, in terms of gradients of the density field. Locally, the fluid satisfies the van der Waals state equation. A finite difference version of LBM is implemented where the relationship $c=\delta s / \delta t$ among the lattice speed $c$ and the space and time steps $\delta s$ and $\delta t$ does no longer hold, as in standard collision - streaming LBM [8-12]. The rejection of this condition has two advantages. First, this allows one to further consider multicomponent fluid systems where the masses of the component particles, as well as the lattice speeds, may be no longer identical [13,14]. Second, higher order numerical schemes (including flux limiter schemes) may be considered in order to reduce unphysical effects like the spurious velocity and the numerical viscosity [7,13-18]. The use of high order numerical schemes in finite difference LBM helps further to improve the numerical stability and accuracy $[7,17]$ while providing a convenient alternative to interpolation supplemented $\operatorname{LBM}[19,20]$.

Our main results is that the sedimentation process induced by gravity is characterized by an exponent $\alpha=1$ independently on the values of viscosity and gravity.

The paper is organized as follows. Our LBM approach is described in Section 2; numerical results are shown in Section 3 and conclusions will be drawn in Section 4.

\section{Description of the model}

In this paper, we use the D2Q9 isothermal finite difference lattice Boltzmann model in two dimensions, which is well known in the literature [9-11,15,21]. This model relies on the following set of $\mathcal{N}=9$ evolution equations for the non-dimensionalized distribution functions $f_{i}(\mathbf{r}, t), i=0,1, \ldots, \mathcal{N}-1$, defined in the nodes $\mathbf{r}=(x, y)$ of a lattice with $\Lambda_{x} \times \Lambda_{y}$ 
nodes $[7-12,18,21,22]$

$$
\begin{aligned}
f_{i}(\mathbf{r}, t+\delta t) & =f_{i}(\mathbf{r}, t)-\delta t \mathbf{e}_{i} \cdot \nabla f_{i}(\mathbf{r}, t)-\frac{\delta t}{\tau}\left[f_{i}(\mathbf{r}, t)-f_{i}^{e q}(\mathbf{r}, t)\right] \\
& +\frac{\delta t}{\chi c^{2}} \mathbf{F} \cdot\left[\mathbf{e}_{i}-\mathbf{u}(\mathbf{r}, t)\right] f_{i}^{e q}(\mathbf{r}, t) .
\end{aligned}
$$

In this non-dimensionalized model, the mass of particles equals 1 . To reduce numerical errors, the second order Monitorized-Central-Difference flux limiter scheme [7,13, 18, 23, 24] was used to calculate the space derivative $\nabla f_{i}(\mathbf{r}, t)$.

The local values of the fluid quantities (particle number density $n$ and velocity $\mathbf{u}$ ) are derived from the distribution functions, as follows

$$
\begin{aligned}
& n(\mathbf{r}, t)=\sum_{i=0}^{\mathcal{N}-1} f_{i}(\mathbf{r}, t), \\
& \mathbf{u}(\mathbf{r}, t)=\frac{1}{n(\mathbf{r}, t)} \sum_{i=0}^{\mathcal{N}-1} f_{i}(\mathbf{r}, t) \mathbf{e}_{i} .
\end{aligned}
$$

The velocity vectors $\left\{\mathbf{e}_{i}\right\}$ are given by

$$
\begin{aligned}
& \mathbf{e}_{0}=0 \\
& \mathbf{e}_{i}=\left(\cos \frac{\pi(i-1)}{2}, \sin \frac{\pi(i-1)}{2}\right) c \quad \text { for } i=1, \ldots, 4 \\
& \mathbf{e}_{i}=\left(\cos \frac{\pi(2 i-9)}{4}, \sin \frac{\pi(2 i-9)}{4}\right) c \sqrt{2} \quad \text { for } \quad i=5, \ldots, 8
\end{aligned}
$$

where $c=c / c_{R}=\sqrt{\theta / \chi}$ is a non-dimensionalized speed, $\theta=T / T_{R}$ is the non-dimensionalized system temperature, and $\chi=1 / 3$. As discussed in [15, 25], the following reference quantities $n_{R}=N_{A} / V_{m c}, T_{R}=T_{c}$ and $c_{R}=\sqrt{k_{B} T_{c} / m_{R}}$, where $N_{A}$ is Avogadro's number, $V_{m c}$ is the molar volume at the critical point of temperature $T_{c}, m_{R}$ is the mass of the fluid particles and $k_{B}$ is Boltzmann's constant, may be used to get the non-dimensionalized values of the particle number density, temperature and speed, respectively. The system size is chosen as reference length $l_{R}$, the reference quantities $t_{R}$ and $a_{R}$ for time and acceleration follow from

$$
\frac{t_{R} c_{R}}{l_{R}}=1 \quad, \quad \frac{a_{R} t_{R}}{c_{R}}=1 .
$$

Since we use finite difference schemes $[7,13,15,22,23]$ to evolve the particle distribution functions according to Eq.(2.1), the lattice spacing $\delta s$ and the time step $\delta s$ are no longer related to the lattice speed $c$ as in standard lattice Boltzmann models [8-11], the temperature $\theta$ is now a control parameter in our simulations. This feature of the finite difference approach allows us to change the system temperature $\theta$ (and hence also the lattice speed $c)$ while preserving the lattice spacing $\delta s$ and the system size in each direction (i.e., the 
corresponding number of lattice nodes). In such models there is more freedom to choose the discrete velocity set, as done recently in a thermal model [26] where the possibility of having different sets of velocities allows to release the constraint of constant temperature.

The equilibrium distribution functions $f_{i}^{e q}=f_{i}^{e q}(\mathbf{r}, t)$ that appear in the evolution equation (2.1) of the D2Q9 model are expressed as series expansion up to second order with respect to the fluid velocity $\mathbf{u}[9-11,21]$

$$
f_{i}^{e q}=w_{i} n\left[1+\frac{\mathbf{e}_{i} \cdot \mathbf{u}}{\chi c^{2}}+\frac{\left(\mathbf{e}_{i} \cdot \mathbf{u}\right)^{2}}{2 \chi^{2} c^{4}}-\frac{(\mathbf{u})^{2}}{2 \chi c^{2}}\right]
$$

where the weight coefficients are

$$
w_{i}=\left\{\begin{aligned}
\frac{4}{9}, & i=0 \\
\frac{1}{9}, & i=1, \ldots 4 \\
\frac{1}{36}, & i=5, \ldots 8
\end{aligned}\right.
$$

The force $\mathbf{F}$ in Eq. (2.1) is introduced to recover the macroscopic equations of an isothermal van der Waals fluid subjected to the gravitational acceleration a. When using the reference pressure $p_{R}=m n_{R} c_{R}^{2}$, this force has the Cartesian components $[7,15,25$, 27-29]

$$
F_{\alpha}=\frac{1}{n} \partial_{\alpha}\left(p^{i d e a l}-p^{w a a l s}\right)+\kappa \partial_{\alpha}\left(\nabla^{2} n\right)+a_{\alpha}
$$

where

$$
p^{i d e a l}=n \theta
$$

is the ideal gas pressure and

$$
p^{w a a l s}=\frac{n \theta}{1-b n}-a n^{2}
$$

is the van der Waals fluid pressure in non-dimensionalized form, $\kappa$ is a constant which controls the surface tension and $\mathbf{a}$ is the gravitational acceleration. The parameters $a$ and $b$ are given by

$$
\begin{aligned}
a & =\frac{9}{8} \frac{\theta_{c}}{n_{c}} \\
b & =\frac{1}{3 n_{c}}
\end{aligned}
$$

where $n_{c}$ is the particle density at the critical temperature $\theta_{c}=1$. In the following we will consider $n_{c}=1$ so the the van der Waals fluid pressure reads

$$
p^{w a a l s}=\frac{3 n \theta}{3-n}-\frac{9}{8} n^{2}
$$


Note that the force (2.8) was already used in $[7,15,18,25]$ to investigate the dynamics and morphology of phase separation in liquid-vapour systems in the absence of gravity $(\mathbf{a}=0)$.

A Chapman-Enskog expansion shows that the continuity and Navier-Stokes equations are recovered in the continuum limit:

$$
\begin{gathered}
\partial_{t} n+\partial_{\beta}\left(n u_{\beta}\right)=0, \\
\partial_{t}\left(n u_{\alpha}\right)+\partial_{\beta}\left(n u_{\alpha} u_{\beta}\right)=-\partial_{\alpha} p^{w a a l s}+\kappa n \partial_{\alpha}\left(\nabla^{2} n\right)+n a_{\alpha}+v \partial_{\beta}\left[n\left(\partial_{\alpha} u_{\beta}+\partial_{\beta} u_{\alpha}\right)\right]
\end{gathered}
$$

with kinematic viscosity $v=\theta \tau$. The terms $-\partial_{\alpha} p^{\text {waals }}+\kappa n \partial_{\alpha}\left(\nabla^{2} n\right)$ at the r.h.s. of the Navier-Stokes equation can also be written in the form $-\partial_{\beta} P_{\alpha \beta}$ where the pressure tensor $P_{\alpha \beta}$ is related to the free energy functional of the van der Waals fluid [30]

$$
\Psi=\int d \mathbf{r}\left[\psi(n, \theta)+\frac{\kappa}{2}(\nabla n)^{2}\right],
$$

$\psi(n, \theta)$ being the bulk free energy density

$$
\psi(n, \theta)=n \theta \ln \left(\frac{3 n}{3-n}\right)-\frac{9}{8} n^{2}
$$

The pressure tensor is [31]

$$
P_{\alpha \beta}=p \delta_{\alpha \beta}+\kappa \partial_{\alpha} n \partial_{\beta} n
$$

with

$$
p=p^{w a a l s}-\kappa n \nabla^{2} n-\frac{\kappa}{2}(\nabla n)^{2}
$$

where $p^{\text {waals }}=n \psi^{\prime}(n)-\psi$ is the equation of state with the critical point at $n_{c}=1, \theta_{c}=1$.

In the sequel, we will consider the case of an acceleration directed upwards: $a_{x}=$ $0, a_{y}=g$. Periodical boundary conditions were considered in the horizontal direction and standard bounce back boundary conditions [8-12] were imposed on top and bottom walls.

\section{Numerical results}

In this Section we report the results of our simulations. For runs we used either a square lattice with $\Lambda_{x}=\Lambda_{y}=1024$ or a rectangular one with $\Lambda_{x}=512$ and $\Lambda_{y}=4096$, lattice spacing $\delta s=1 / 256$ and time step $\delta t=10^{-5}$, as in [7]. All quenches below the critical temperature $\theta_{c}=1$ were to the temperature $\theta=0.79$ where the coexisting densities are $n_{\text {liquid }}=1.956$ and $n_{\text {vapor }}=0.226$. Each simulation was started with small fluctuations $(0.1 \%)$ in the density about a mean value $\hat{n}$ that was either symmetric $(\hat{n}=1.09$, liquid fraction $\beta=0.5)$ or slightly off-symmetric $(\hat{n}=1.0, \beta=0.45)$. The parameter $\kappa$ controlling the surface tension was set to $5 \times 10^{-6}$ to have an interface thickness of $\sim 6$ lattice spacings. The corresponding value of the surface tension $\sigma$ was evaluated by using the Laplace law 
[25] and a value $\sigma=2.0 \times 10^{-3}$ was measured. The value of the constant $g$ controlling the external acceleration, was varied in the range $[0.0001,0.005]$. Results were not dependent on its specific value as shown in the following. The viscosity was varied by changing $\tau$. We used the values $\tau=10^{-4}$ and $\tau=10^{-3}$, which allow us to access low and high viscosity regimes, respectively, as shown in a previous work where the present model without gravity was used to study the phase separation in liquid-gas systems [7].

The process of phase separation depends on the interplay among three main driving forces: The viscous one $F_{v}=n v l_{R} c_{R}$, the gravity one $F_{g}=n g l_{R}^{3}$, and the surface tension one $F_{S}=\sigma l_{R}$. It may be then useful to evaluate their relative contributions introducing the Bond number $B o=\frac{F_{g}}{F_{S}}=\frac{n g l_{R}^{2}}{\sigma}$, the Capillary number $C a=\frac{F_{v}}{F_{S}}=\frac{n v c_{R}}{\sigma}$, and the ratio $\frac{B o}{C a}=$ $\frac{F_{g}}{F_{v}}=\frac{g l_{R}^{2}}{v c_{R}}$. The choice of the input parameters is such to access the ranges $0.05 \leq B o \leq 2.5$, $0.04 \leq \mathrm{C} a \leq 0.4$, and $0.13 \leq B o / C a \leq 65$.

After the initial stages when the mixture starts to phase separate, the effect of the gravitational force is to accumulate material at walls: The heavy phase (liquid) is moved to the top wall and the light phase (vapor) stays at the bottom wall. The evolution of domains in the cases at high $\left(\tau=10^{-3}\right)$ and low $\left(\tau=10^{-4}\right)$ viscosities is shown in Figs. 1 1 , 2 for $g=0.005$. At intermediate times between $t \simeq 3$ and $t \simeq 10$, anisotropic patterns can be observed in the bulk region far from the walls, with domains slightly elongated along the vertical direction. The main difference that can be observed between the two figures is the presence of many droplets in the case at high viscosity as compared to the case at low viscosity. The reason is due to the fact that in the latter case hydrodynamics is effective in coalescing droplets, thus producing a more homogeneous pattern.

In order to gain some insight into the law governing the accumulation of material at walls, we measured the average thickness $L$ of layers adjacent to the walls. For each column of the lattice we looked for all the sites next to the walls where there was an interface between the liquid and vapor phases. To be more specific we found all the lattice sites along the $x$-direction with the smallest distance $y_{b}^{*}(x)$ to the bottom wall such that $\left[n\left(x, y_{b}^{*}(x)\right)-\hat{n}\right]\left[n\left(x, y_{b}^{*}(x)+1\right)-\hat{n}\right]<0$ with $y_{b}^{*}(x)<\Lambda_{y} / 2$ and all the sites with the smallest distance $\Lambda_{y}-y_{t}^{*}(x)$ to the top wall such that $\left[n\left(x, y_{t}^{*}(x)\right)-\hat{n}\right]\left[n\left(x, y_{t}^{*}(x)-1\right)-\hat{n}\right]<0$ with $y_{t}^{*}(x)>\Lambda_{y} / 2$. We defined

$$
L=\frac{1}{2 \Lambda_{x}} \sum_{x=1}^{\Lambda_{x}}\left[y_{b}^{*}(x)+\left(\Lambda_{y}-y_{t}^{*}(x)\right)\right]<\frac{\Lambda_{y}}{2}
$$

The time evolution of $L$ is reported in Fig. 3 for different values of $\tau$ and $g$. We have a clear and convincing indication that in all the cases the growth is consistent with a power law with growth exponent $\alpha=1$ which depends neither on $g$ nor on $\tau$. The growth is observed over almost two time decades until the system is entirely separated in two parts of different composition. In particular, we want to stress the fact that the exponent $\alpha=1$ is observed in both cases, when the gravitational force $F_{g}$ is small compared to the 

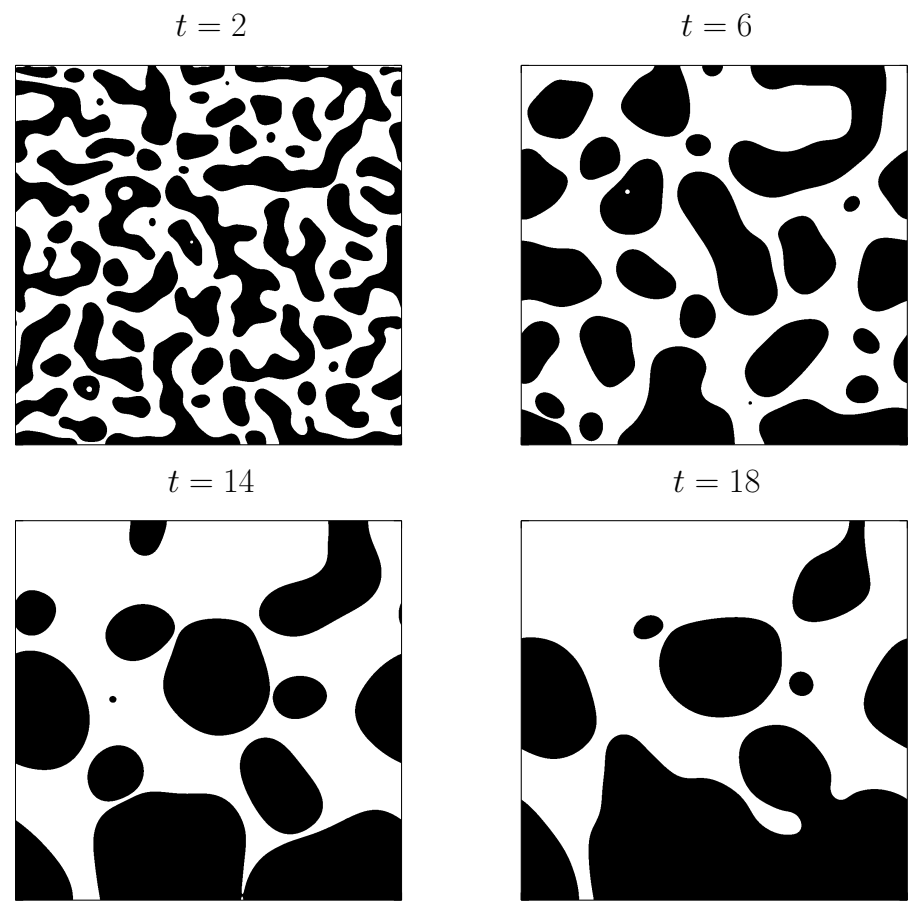

$t=22$

$$
t=26
$$
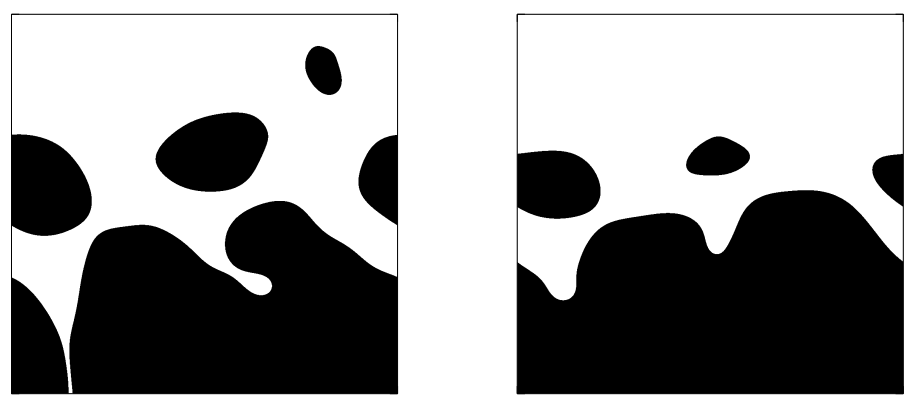

Figure 1: Contour plots of the density $n$ in the case with $\tau=10^{-3}, g=0.005$ and $\beta=0.5$. Color code: white/black $\rightarrow$ liquid/vapor. 

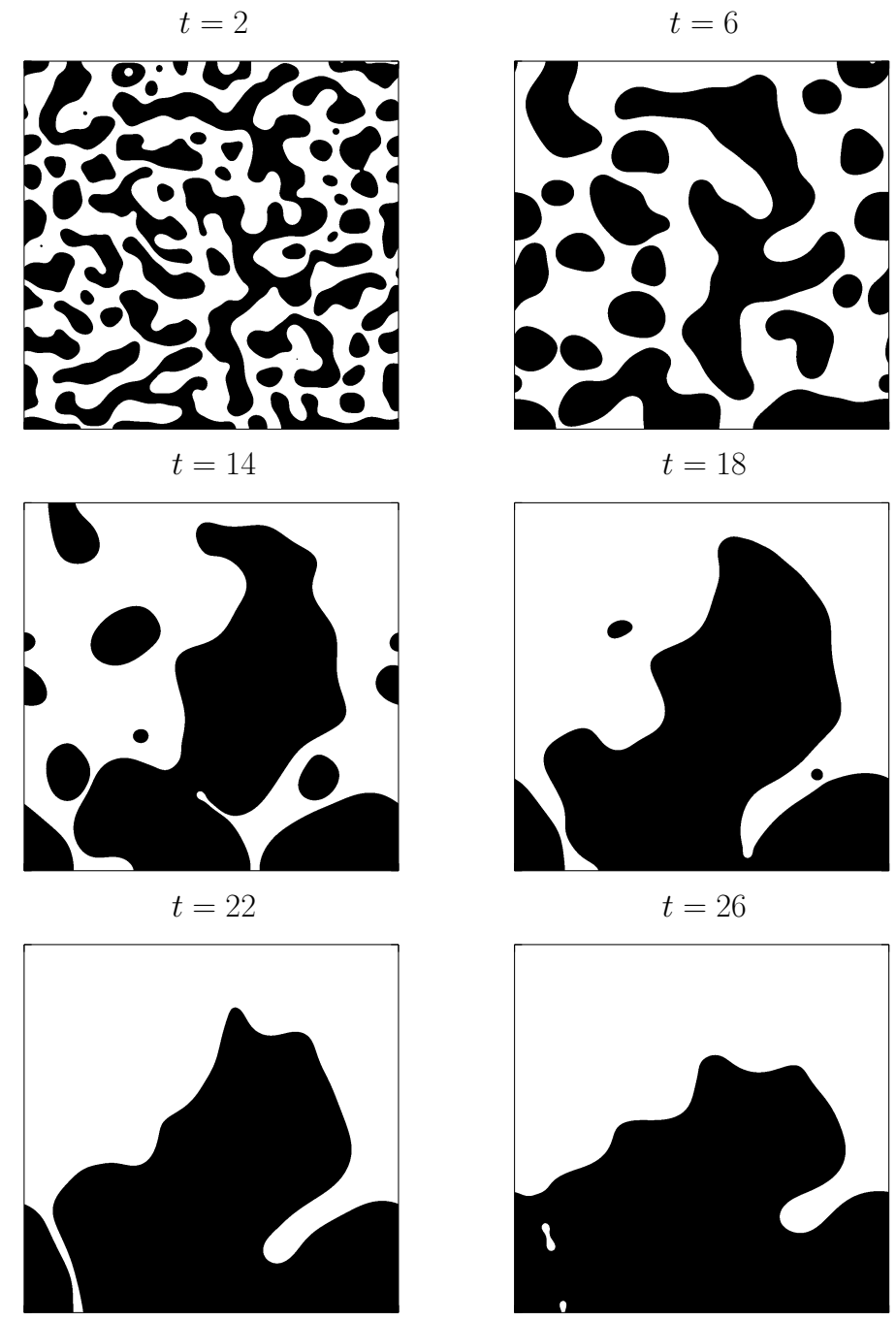

Figure 2: Contour plots of the density $n$ in the case with $\tau=10^{-4}, g=0.005$ and $\beta=0.5$. Color code: white/black $\rightarrow$ liquid/vapor. 


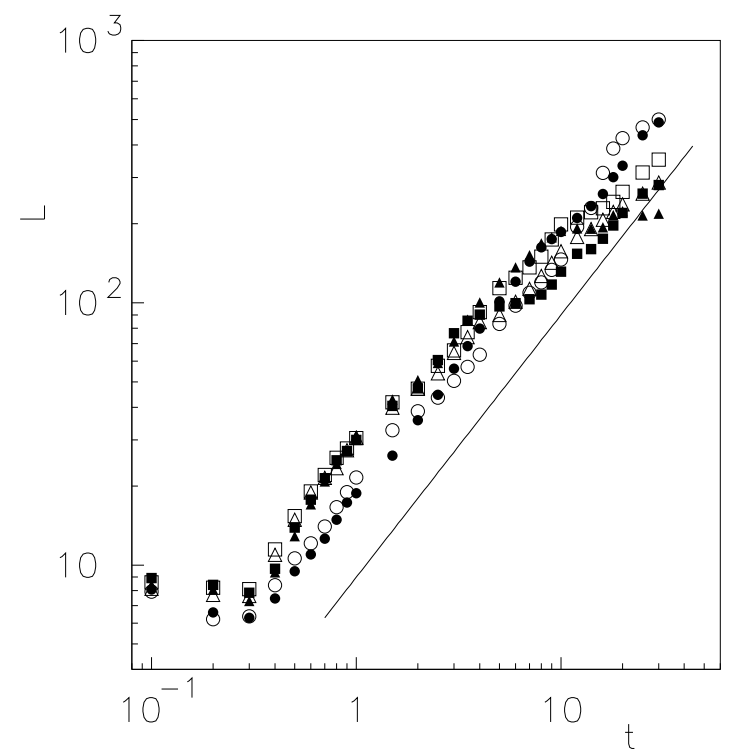

Figure 3: Time evolution of the average thickness of layers at walls in the cases with $g=$ $0.0001(\triangle), 0.001(\square), 0.005(\circ)$ for $\tau=10^{-3}$ (filled symbols), $10^{-4}$ (empty symbols) and $\beta=0.5$. The solid line is a guide to the eye and has slope 1 .

surface tension force $F_{S}(B o<1)$, as well as when $F_{g}$ is small compared to the viscous one $F_{v}(B o / C a<1)$. This indicates that the existence of the same scaling exponent $\alpha$ in the gravity direction is exclusively due to the presence of the gravity force $F_{g}>0$. Our result is in agreement with previous studies of mixtures where hydrodynamics was neglected [6,32-34]. In another study on the phase separation of binary fluids [35] where hydrodynamic effects were considered, it was argued that the growth exponent is $\alpha=$ $0.6 \pm 0.1$ and is not affected by the presence of gravity. The present study shows that the value of the growth exponent is independent on the value of the viscosity and of the gravity. Similar results were obtained when considering the case of a slightly offsymmetric mixtures with $\beta=0.45$.

In order to better characterize the morphology of domains we simulated the behaviour of a very large system with symmetric composition $(\beta=0.5)$ and size $512 \times 4096$, for $g=0.005$ and $\tau \in\left\{10^{-4}, 10^{-3}\right\}$. Also in this case we found that $L$ grows with the exponent 1. In order to estimate the domains size in the two spatial directions, due to the anisotropy induced by gravity, we computed the inverse of the first moment of the structure factor $[36,37]$

$$
R_{x}(t)=\pi \frac{\int d \mathbf{k} C(\mathbf{k}, t)}{\int d \mathbf{k} k_{x} C(\mathbf{k}, t)}
$$



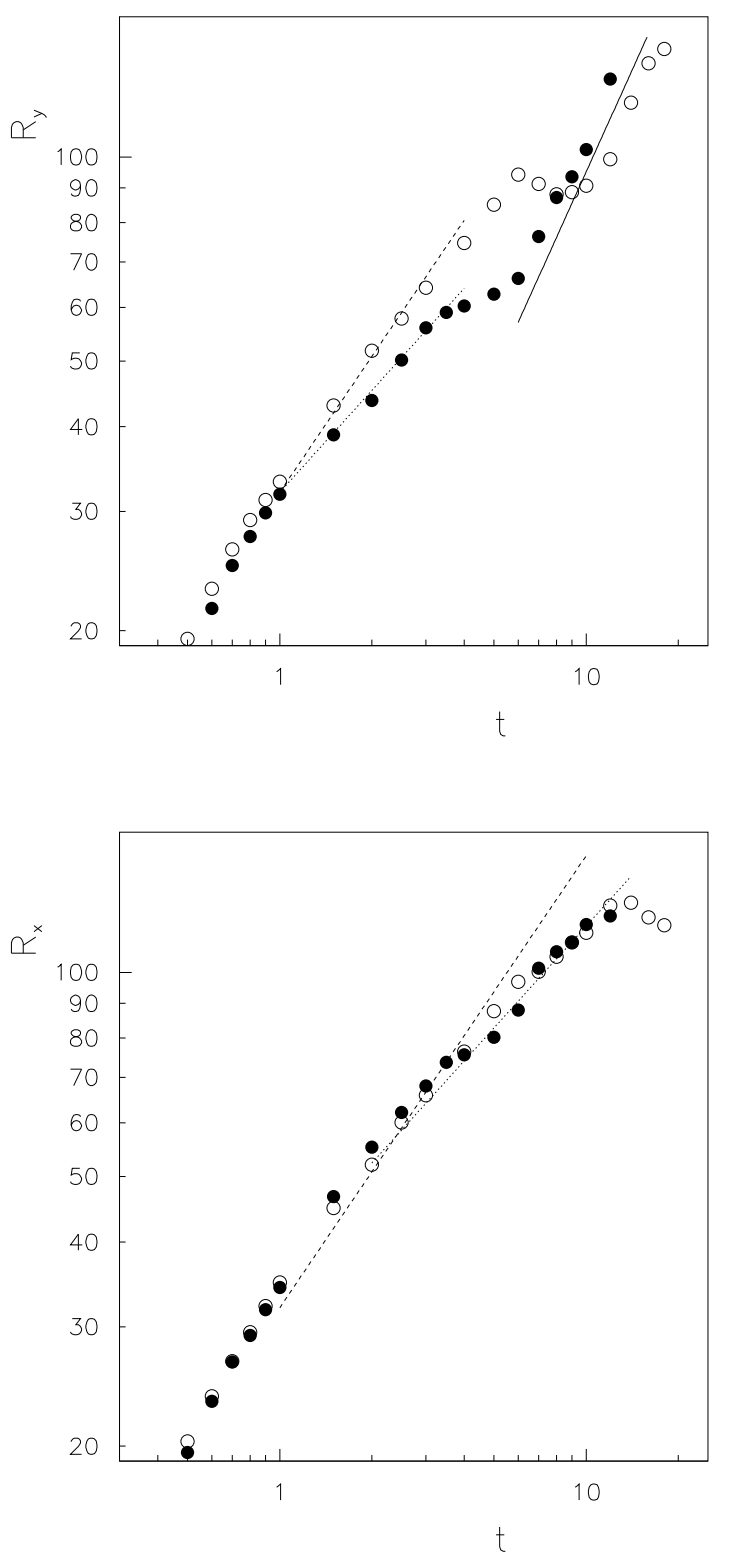

Figure 4: Time evolution of the size of domains $R_{y}$ (upper panel) and $R_{x}$ (lower panel) in the case with $g=0.005$ for $\tau=10^{-3}(\bullet), 10^{-4}(\circ)$ and $\beta=0.5$ on a lattice $512 \times 4096$. The lines serve as eyeguide and have the slopes $1 / 2$ (dotted line), 2/3 (dashed line), and 1 (full line). 
and similarly for the $y$ direction where

$$
C(\mathbf{k}, t)=<\tilde{n}(\mathbf{k}, t) \tilde{n}(-\mathbf{k}, t)>
$$

with $\tilde{n}(\mathbf{k}, t)$ the spatial Fourier transform of $n(\mathbf{r}, t)-\hat{n}$. The results are shown in Fig. 4. Along the gravity direction, we see that $R_{y}$ starts to grow with the same exponents as in the case without gravity: $1 / 2$ for $\tau=0.001$ and $2 / 3$ for $\tau=0.0001$ [7]. The transition to a regime consistent with the growth exponent 1 is observed at later times. Along the horizontal direction, we get $R_{x} \sim t^{1 / 2}$ in the high viscosity case $(\tau=0.001)$, a behaviour that is similar to the case when the system is subjected to no gravity. However, at low viscosity $(\tau=0.0001)$ the growth exponent along the horizontal direction in the presence of gravity is smaller than the expected value $2 / 3$, which is achieved without gravity. It is interesting to note that similar conclusions were drawn in the case of phase separation of binary mixtures under gravity [38]. In their study, the authors observed that in the diffusive and viscous regimes the growth exponent is always equal to 1 along the vertical (gravity) direction, while in the horizontal direction there is a slowing down of the growth rate with respect to the case without gravity [38]. In order to better elucidate these features one would need to perform higher resolution simulations to access a wider range of length scales.

\section{Conclusions}

In this paper we have introduced an external gravitational force in an isothermal lattice Boltzmann model for the van der Waals fluid. We have studied phase separation in systems with different viscosities and various values of the gravitational acceleration. In the absence of gravity, the growth exponent is known to have specific values [7], which depend on the fluid viscosity. When the liquid-vapor system was subjected to the gravitational force, we measured the evolution of the characteristic size (along the gravity direction) of the growing domains and found the same exponent $\alpha=1$ for all the cases considered, even if the fluid viscosity and the gravitational acceleration were different. Further extension of our parallel computing code to three dimensions would allow to evaluate the growth exponents in a more realistic case.

\section{Acknowledgments}

The authors acknowledge support from CEEX 11 (2005-2008), as well as CNR-INFM for a grant at CINECA Consortium for Supercomputing.

\section{References}

[1] A. J. Bray, Theory of phase-ordering kinetics, Advances in Physics 43 (1994) 357 - 459. 
[2] J. M. Yeomans, Phase ordering in Fluids, in Annual Reviews of Computational Physics VII, pp. 61 - 84 (D.Stauffer, Editor), World Scientific, Singapore, (2000).

[3] A. Onuki, Phase transitions of fluids in shear flow, Journal of Physics - Condensed Matter 9 (1997) 6119 - 6157.

[4] V. M. Kendon, M. E. Cates, I. Pagonabarraga, J.-C. Desplat, and P. Bladon, Inertial effects in three-dimensional spinodal decomposition of a symmetric binary fluid mixture: a lattice Boltzmann study, Journal of Fluid Mechanics 440 (2001) 147 - 203.

[5] C. K. Chan and W. I. Goldburg, Late-stage phase separation and hydrodynamic flow in a binary liquid mixture, Physical Review Letters 58 (1987) 674 -677.

[6] S. Puri, K. Binder, and S. Dattagupta, Dynamical scaling in anisotropic phase-separating systems in a gravitational field, Physical Review B 46 (1992) 98 - 105.

[7] V. Sofonea, A. Lamura, G. Gonnella, and A. Cristea, Finite-difference lattice Boltzmann model with flux limiters for liquid-vapor systems, Physical Review E 70 (2004) 046702.

[8] R. Benzi, S. Succi, and M. Vergassola, The lattice Boltzmann equation: theory and applications, Physics Reports 222 (1992) 145 - 197.

[9] S. Chen and G. D. Doolen, Lattice Boltzmann Method for fluid Flows, Annual Review of Fluid Mechanics 30 (1998) 329 - 364.

[10] D. A. Wolf-Gladrow, Lattice Gas Cellular Automata and Lattice Boltzmann Models, Springer, Berlin, 2000.

[11] S. Succi, The Lattice Boltzmann Equation for Fluid Dynamics and Beyond, Clarendon Press, Oxford, 2001.

[12] B. Duenweg and A. J. C. Ladd, Lattice Boltzmann simulations of soft matter systems, in Advences in Polymer Science 221 (2009) 89 - 166.

[13] A. Cristea and V. Sofonea, Two component lattice Boltzmann model with flux limiters, Central European Journal of Physics 2 (2004) 382 - 396.

[14] V.Sofonea, Discontinuous Galerkin schemes for isothermal lattice Boltzmann models in one dimension, International Journal of Modern Physics C 19 (2008) 677 - 688.

[15] A. Cristea and V. Sofonea, Reduction of spurious velocity in finite difference lattice Boltzmann models for liquid-vapor systems, International Journal of Modern Physics C 14 (2003) $1251-1266$.

[16] V. Sofonea and R. F. Sekerka, Viscosity of finite difference lattice Boltzmann models, Journal of Computational Physics 184 (2003) 422 - 434.

[17] V. Sofonea and R. F. Sekerka, Diffusivity of two-component isothermal finite difference lattice Boltzmann models, International Journal of Modern Physics C 16 (2005) 1075 - 1090.

[18] A. Cristea, G. Gonnella, A. Lamura, and V. Sofonea, Finite-difference lattice Boltzmann model for liquid-vapor systems, Mathematics and Computers in Simulation 72 (2006) 113 $-116$.

[19] X. He, L. Luo, and M. Dembo, Some progress in lattice Boltzmann method. part I. Nonuniform mesh grids, Journal of Computational Physics 129 (1996) 357 - 363.

[20] X. He, Error analysis for the interpolation-supplemented lattice-Boltzmann equation scheme, International Journal of Modern Physics C 8 (1997) 737 - 745.

[21] Y. H. Qian, D. d'Humières, and P. Lallemand, Lattice BGK Models for Navier-Stokes Equation, Europhysics Letters 17 (1992) 479 - 484.

[22] N. Cao, S. Chen, S. Jin, and D. Martínez, Physical symmetry and lattice symmetry in the lattice Boltzmann method, Physical Review E 55 (1997) R21 - R24.

[23] R. J. LeVeque, Numerical Methods for Conservation Laws, Birkhäuser, Basel, 1992.

[24] G. Gonnella, A. Lamura, and V. Sofonea, Lattice Boltzmann simulation of thermal nonideal 
fluids, Physical Review E 76 (2007) 036703.

[25] A. Cristea, Numerical effects in a finite difference lattice Boltzmann model for liquid-vapour systems, International Journal of Modern Physics C 17 (2006) 1191 - 1201.

[26] M. Watari and M. Tsutahara, Two-dimensional thermal model of the finite-difference lattice Boltzmann method with high spatial isotropy, Physical Review E 67 (2003) 036306.

[27] X.Y.He, X.W.Shan and G.D.Doolen, Discrete Boltzmann equation for nonideal gases, Physical Review E 57 (1998) R13 - R16.

[28] X. Y. He, S. Y. Chen and R. Y. Zhang, A lattice Boltzmann scheme for incompressible multiphase flow and its applications in simulation of Rayleigh - Taylor instability, Journal of Computational Physics 152 (1999) 642 - 663.

[29] X. Y. He and G. D. Doolen, Thermodynamic foundations of kinetic theory and lattice Boltzmann models for multiphase flows, Journal of Statistical Physics 107 (2002) 309 - 328.

[30] J. S. Rowlinson and B. Widom, Molecular Theory of Capillarity, Clarendon Press, Oxford, 1982.

[31] R. Evans, The nature of the liquid-vapour interface and other topics in the statistical mechanics of non-uniform, classical fluids, Advances in Physics 28 (1979) 143 - 200.

[32] A. M. Lacasta, A. Hernandez-Machado, and J. M. Sancho, Front and domain growth in the presence of gravity, Physical Review B 48 (1993) 9418 - 9425.

[33] S. Puri, N. Parekh, and S. Dattagupta, Phase ordering dynamics in a gravitational field, Journal of Statistical Physics 75 (1994) 839 - 857.

[34] S. Puri, Phase separation kinetics in anisotropic systems, Physica A 224 (1996) 101 - 112.

[35] W. Ma, A. Maritan, J. R. Banavar, and J. Koplik, Dynamics of phase separation of binary fluids, Physical Review A 45 (1992) R5347 - R5350.

[36] F. Corberi, G. Gonnella, and A. Lamura, Spinodal decomposition of binary mixtures in uniform shear flow, Physical Review Letters 81 (1998) 3852 - 3855.

[37] F. Corberi, G. Gonnella, and A. Lamura, Two-scale competition in phase separation with shear, Physical Review Letters 83 (1999) 4057 - 4060.

[38] V. E. Badalassi, H. D. Ceniceros and S. Banerjee, Gravitational effects on structure development in quenched complex fluids, Annals of the New York Academy of Sciences 1027 (2004) 371 - 382. 